\title{
Aplicabilidad del Ages \& Stages Questionnaires para el tamizaje del desarrollo psicomotor
}

\author{
LUISA SCHONHAUT B. ${ }^{1}$, IVÁN ARMIJO R., ${ }^{2,3}$ \\ 1. Departamento de Pediatría, Facultad de Medicina Clínica Alemana, Universidad del Desarrollo. \\ 2. Facultad de Psicología Universidad del Desarrollo. \\ 3. Facultad de Psicología Universidad Gabriela Mistral.
}

\section{ABSTRACT \\ Applicability of the Ages \& Stages Questionnaires (ASQ) as a developmental screening tool for psychomotor delay}

The screening and timely intervention of developmental psychomotor delay (DSM) has proven benefits for the child, families and society. In order to improve the efficiency in the identification of these disorders, many scientific groups recommend performing clinical monitoring during health controls and the use of standard evaluations at specific patient age or when delays are suspected. In recent years, screening instruments have been developed based on the report of parents or primary caregivers, which have the advantage of being inexpensive, require little application and interpretation time and involve the adults who are responsible for the care and stimulation of the child. One of these tools is the Ages and Stages Questionnaires (ASQ), which has been widely used in the US and validated by several countries. This study describes the DSM assessment practices used in Chile and backs up the use of monitoring systems based on the report of parents/caregivers. ASQ validation experiences in other countries are discussed along with their psychometric characteristics for the Chilean population and consequent applicability.

(Key words: Motor development, screening, Ages and Stages Questionnaires).

Rev Chil Pediatr 2014; 85 (1): 12-21

\section{RESUMEN}

La pesquisa e intervención oportunas de los trastornos del desarrollo psicomotor (DSM), tiene demostrados beneficios para el niño, su familia y la Sociedad. Con la finalidad de mejorar la eficacia en la identificación de las dificultades del DSM, distintas sociedades científicas recomiendan realizar una vigilancia clínica del desarrollo en los controles de salud y aplicar evaluaciones estandarizadas a edades específicas o frente a la sospecha de déficit. En los últimos años se han desarrollado instrumentos de tamizaje basados en el reporte de padres o cuidadores principales, los que cuentan con la ventaja de ser de bajo costo, demandan poco tiempo en su aplicación e interpretación e involucran a los adultos responsables del cuidado y estimulación del niño.

Recibido el 27 de enero de 2014, aceptado para publicación el 03 de febrero de 2014.

Este trabajo cumple con los requisitos sobre consentimiento /asentimiento informado, comité de ética, financiamiento, estudios animales y sobre la ausencia de conflictos de intereses según corresponda.

Correspondencia a:

Luisa Schonhaut Berman

E-mail: Ischonhaut@alemana.c 
Uno de estos instrumentos, el Ages and Stages Questionnaires (ASQ) ha sido ampliamente utilizado en USA y validado en varios países. En el presente manuscrito se describen las prácticas de evaluación del DSM que se utilizan en Chile y se fundamenta la implementación de un sistema de monitorización del desarrollo infantil basado en el reporte de padres/cuidadores a nivel nacional. Se revisan experiencias de validación del ASQ en otros países y sus características psicométricas para la población Chilena y las implicancias de su aplicabilidad clínica.

(Palabras clave: Desarrollo psicomotor, tamizaje, Ages and Stages Questionnaires).

Rev Chil Pediatr 2014; 85 (1): 12-21

\section{Introducción}

El déficit del desarrollo psicomotor (DSM) y la discapacidad tienen un importante impacto en la sociedad en términos de costos en salud, soporte educacional y servicios de apoyo ${ }^{1,2}$. Ha sido ampliamente demostrado que la pesquisa precoz de los niños con déficit permite una intervención oportuna y efectiva ${ }^{3,4}$. No obstante la evidencia, la identificación de los niños con dificultades más sutiles del DSM representa un verdadero desafío para los pediatras ${ }^{5}$. Distintos estudios han demostrado que la impresión clínica es subjetiva y poco sensible para este $\mathrm{fin}^{6}$, por este motivo, se recomienda la aplicación de escalas de tamizaje estandarizadas a edades específicas o frente a factores de riesgo ${ }^{7}$.

A nivel mundial se han desarrollado distintas pruebas de tamizaje del DSM, entre las que destacan las de reporte de padres o cuidadores, las que cumplen con los valores de sensibilidad y especificidad recomendadas por la Academia Americana de Pediatría (AAP) ${ }^{7}$. Una de las escalas más ampliamente utilizada es el Ages and Stages Questionnaires (ASQ), desarrollada en la Universidad de Oregon en los años ochenta, posteriormente actualizada y validada en varios países ${ }^{8,9}$.

El objetivo del presente manuscrito es revisar experiencias de validación del ASQ y fundamentar la importancia de su implementación a nivel nacional en la actualidad.

\section{Repensando la evaluación del DSM}

En Chile desde 1990 existe un Programa de Estimulación y Evaluación del DSM, el cual cubre a los menores que se atienden en el sis- tema público de salud. Este programa considera la evaluación de los niños mediante dos pruebas desarrolladas en Chile en la década del 70-80: la Escala de Evaluación del Desarrollo Psicomotor (EEDP) y el Test de Desarrollo Psicomotor (TEPSI). Dichas escalas son aplicadas por enfermera $u$ otro profesional capacitado, en los controles de salud de los 8,18 y 36 meses $^{10}$; además del inconveniente relacionado con el consumo de tiempo profesional y de la exposición del niño a una "situación extraña", tienen la desventaja de carecer de validez y actualización ${ }^{11,12}$. De acuerdo al registro del sistema público de salud de Chile 2013, $11,6 \%$ de los niños tendrían riesgo o retraso en el $\mathrm{DSM}^{13}$. Esta prevalencia es inferior a la reportada tanto nacional ${ }^{14,15}$ como internacionalmente $^{16}$, lo que sugiere que las estrategias actualmente implementadas en atención primaria podrían estar sub-identificando dificultades en los niños evaluados.

Por otro lado, en los controles de supervisión de salud del sector privado, es el pediatra quien realiza una vigilancia del DSM basada en criterios clínicos con énfasis en la identificación de factores de riesgo y logro de los hitos esperados para la edad, sumado a la apreciación clínica. No obstante, se ha evidenciado que dicha estrategia es subjetiva y poco confiables para pesquisar dificultades más sutiles ${ }^{6,17}$. Por este motivo se recomienda complementar la evaluación clínica con pruebas de tamizaje estandarizadas a edades específicas o frente a factores de riesgo ${ }^{7,18,19}$. Cuando un niño fracasa en las pruebas de tamizaje, debería ser derivado a una evaluación diagnóstica integral, para decidir su incorporación en un programa de apoyo $^{20,21}$. En la tabla 1 se sintetizan las distintas etapas de la evaluación del DSM. 
Tabla 1. Proceso de pesquisa de dificultades del Desarrollo Psicomotor ${ }^{20,21}$

\begin{tabular}{|c|c|c|c|}
\hline Procedimiento & Grupo blanco & Objetivos & Métodos \\
\hline $\begin{array}{l}\text { Vigilancia clínica del } \\
\text { desarrollo psicomotor }\end{array}$ & $\begin{array}{l}\text { Todos los niños en los } \\
\text { controles de supervisión } \\
\text { de salud }\end{array}$ & $\begin{array}{l}\text { Promoción de la salud y el desarro- } \\
\text { llo, del cuidado paterno, identificar } \\
\text { factores de riesgo y elementos de } \\
\text { sospecha de dificultades del DSM }\end{array}$ & $\begin{array}{l}\text { Pesquisa basada en el Juicio clínico } \\
\text { del profesional de salud y análisis del } \\
\text { logro de hitos del desarrollo, sin em- } \\
\text { plear pruebas estandarizadas }\end{array}$ \\
\hline Screening o tamizaje & $\begin{array}{l}\text { Todos los niños a edades } \\
\text { específicas o frente a la } \\
\text { sospecha de déficit }\end{array}$ & $\begin{array}{l}\text { Identificar aquellos niños que debe- } \\
\text { rían recibir una evaluación diagnós- } \\
\text { tica más especializada, verificar las } \\
\text { preocupaciones de los padres }\end{array}$ & $\begin{array}{l}\text { Empleo de pruebas estandarizadas } \\
\text { para pesquisar aquellos niños en } \\
\text { riesgo de déficit del desarrollo }\end{array}$ \\
\hline Examen de desarrollo & $\begin{array}{l}\text { Niños con sospecha clínica } \\
\text { de déficit }\end{array}$ & $\begin{array}{l}\text { Confirmar la sospecha y definir plan } \\
\text { de estudio específico }\end{array}$ & $\begin{array}{l}\text { Evaluación clínica especializada y } \\
\text { examen físico completo }\end{array}$ \\
\hline Pruebas diagnósticas & $\begin{array}{l}\text { Niños con un tamizaje } \\
\text { alterado }\end{array}$ & $\begin{array}{l}\text { Proveer una detallada descripción } \\
\text { y categorización del desarrollo del } \\
\text { niño para establecer plan de manejo } \\
\text { y seguimiento }\end{array}$ & $\begin{array}{l}\text { Herramientas estandarizadas de eva- } \\
\text { luación y diagnóstico de las dificulta- } \\
\text { des del DSM, que varían en cada país } \\
\text { y según la edad de los niños }\end{array}$ \\
\hline
\end{tabular}

Estudios muestran que, a pesar de existir políticas de supervisión de salud que enfatizan la importancia pruebas de tamizaje estandarizadas para la evaluación del $\operatorname{DSM}^{7}$, no más del $50 \%$ de los pediatras utiliza rutinariamente ${ }^{22,23}$. Las barreras descritas para ello son el tiempo que demanda su correcta aplicación, su costo, desconfianza en los test, falta de entrenamiento o de claridad respecto a los procesos de derivación ${ }^{24}$.

\section{Nuevas alternativas para el tamizaje del DSM}

Internacionalmente se han validado distintos instrumentos para el tamizaje del DSM, los que cumplen con los requisitos de poseer adecuadas propiedades psicométricas, ser confiables y reproducibles. Uno de los debates en la actualidad está centrado en cuál prueba elegir. La AAP no se pronuncia por ningún instrumento en particular, dado las diferentes propiedades y énfasis que caracterizan los test disponibles y las distintas modalidades de aplicación ${ }^{7}$.

Las pruebas basadas en el reporte de padres o cuidadores principales han ganado cada vez mayor difusión dado su bajo costo, el poco tiempo que demandan y el involucramiento de los adultos responsables del cuidado y estimulación del niños ${ }^{25-27}$. Además, han demostrado ser altamente confiables, siendo utilizados incluso en el seguimiento de niños con factores de riesgo biológico ${ }^{28-30}$. Entro estas, las escalas que han ganado mayor popularidad son el ASQ y el Parents' Evaluation of Developmental Status (PEDS) ${ }^{23}$.

Las diferencias entre ambas evaluaciones radican en que, mientras el PEDS está enfocado a obtener la preocupación de los padres a través de preguntas abiertas ${ }^{31}$, el ASQ indaga por el logro de habilidades observables, a través de preguntas estructuradas y concretas?. En un estudio en que se compraron ambos cuestionarios con un estándar de referencia, Limbos encontró que el ASQ era más sensible y específico para la identificación de déficits del DSM ${ }^{32}$.

El ASQ consiste en una serie de 21 cuestionarios para niños de 2 meses hasta los 5 años. Cada cuestionario consta de 5 dominios, de 6 preguntas cada una, que evalúan diferentes ámbitos del DSM, como son Comunicación, Motricidad fina, Motricidad gruesa, Resolución de problemas y Relaciones interpersonales. Cada pregunta se responde con las alternativas "sí" (10 puntos), "ocasionalmente" (5 puntos) o "todavía no" (0 puntos), los puntajes de cada dominio se suman, teniendo un total posible de 60 puntos por área. Al final hay una sección de 7 preguntas abiertas destinadas a indagar posibles preocupaciones de los padres.

El ASQ se ha adaptado y validado en Taiwan, Noruega, Holanda, Turquía, India, Irán y Brasil, entre otros países; las publicaciones relacionadas con los procesos de vali- 
Tabla 2. Comparación entre estudios de validación del ASQ en distintos países

\begin{tabular}{|c|c|c|c|c|c|c|}
\hline País & Autor & $\begin{array}{c}\text { Año } \\
\text { publicación }\end{array}$ & $\begin{array}{l}\text { n y características } \\
\text { de los niños }\end{array}$ & $\begin{array}{l}\text { Edad } \\
\text { aplicación } \\
\text { ASQ }\end{array}$ & $\begin{array}{l}\text { Evaluación de } \\
\text { confiabilidad }\end{array}$ & $\begin{array}{l}\text { Evaluación de } \\
\text { validez }\end{array}$ \\
\hline USA & $\begin{array}{l}\text { Squires \& } \\
\text { Bricker }^{8,9}\end{array}$ & $\begin{array}{l}1999 \\
2009\end{array}$ & 18.572 niños & $\begin{array}{l}2-60 \\
\text { meses }\end{array}$ & $\begin{array}{l}\text { Consistencia interna } \\
\text { Estabilidad temporal } \\
\text { Acuerdo interjuez }\end{array}$ & Validez de concurrente \\
\hline Taiwan & Tsai y cols ${ }^{33}$ & 2006 & $\begin{array}{l}101 \text { niños sanos } \\
10 \text { niños con } \\
\text { discapacidad }\end{array}$ & 36 meses & $\begin{array}{l}\text { Consistencia interna, } \\
\text { confiabilidad interjuez }\end{array}$ & $\begin{array}{l}\text { Validez de criterio y de } \\
\text { contenido }\end{array}$ \\
\hline Noruega & $\begin{array}{l}\text { Richter \& } \\
\text { Janson }{ }^{34}\end{array}$ & 2007 & 1.172 niños & $\begin{array}{l}4 \text { a } 60 \\
\text { meses }\end{array}$ & $N D^{*}$ & Validez de constructo \\
\hline Corea & Heo y cols $^{35}$ & 2008 & 3.220 niños & $\begin{array}{l}4 \text { a } 60 \\
\text { meses }\end{array}$ & Consistencia interna & $\begin{array}{l}\text { Validez concurrente. } \\
\text { Análisis Rasch que } \\
\text { incluye pruebas al ítem }\end{array}$ \\
\hline Holanda & $\begin{array}{l}\text { Kerstjens y } \\
\text { cols }^{36} \\
\text { Hornman y } \\
\text { cols }^{37}\end{array}$ & $\begin{array}{l}2009 \\
2013\end{array}$ & $\begin{array}{l}562 \text { RNT } \\
1.510 \text { prematuros } \\
394 \text { RNT } \\
1063 \text { prematuros }\end{array}$ & $\begin{array}{l}48 \text { meses } \\
60 \text { meses }\end{array}$ & Consistencia interna & $\begin{array}{l}\text { Validez de constructo } \\
\text { Validez predictiva }\end{array}$ \\
\hline Turquía & $\begin{array}{l}\text { Kapci y } \\
\text { cols }^{38}\end{array}$ & 2010 & $\begin{array}{l}375 \text { niños con FR } \\
564 \text { sanos } \\
39 \text { con discapacidad }\end{array}$ & $\begin{array}{l}3 \text { a } 72 \\
\text { meses }\end{array}$ & $\begin{array}{l}\text { Estabilidad temporal } \\
\text { Acuerdo interjuez }\end{array}$ & Validez concurrente \\
\hline India & $\begin{array}{l}\text { Juneja y } \\
\text { cols }^{39}\end{array}$ & 2011 & $\begin{array}{l}120 \text { niños sin FR } \\
80 \text { con FR }\end{array}$ & $\begin{array}{c}4,10,18 \\
24\end{array}$ & $N D^{*}$ & Validez concurrente \\
\hline Irán & $\begin{array}{l}\text { Vameghi y } \\
\text { cols }^{40}\end{array}$ & 2013 & 10.516 niños & $\begin{array}{l}4 \text { a } 60 \\
\text { meses }\end{array}$ & Consistencia interna & $\begin{array}{l}\text { Validez de constructo } \\
\text { usando análisis factorial }\end{array}$ \\
\hline Brasil & $\begin{array}{l}\text { Filgueiras y } \\
\text { cols }^{41}\end{array}$ & 2013 & $\begin{array}{l}45,640 \text { niños asistentes } \\
\text { a } 468 \text { públicos** }\end{array}$ & $\begin{array}{l}6 \text { a } 60 \\
\text { meses }\end{array}$ & Consistencia interna & $\begin{array}{l}\text { Análisis factorial } \\
\text { exploratorio }\end{array}$ \\
\hline Chile & $\begin{array}{l}\text { Armijo y } \\
\text { Cols }^{46}\end{array}$ & 2014 & $\begin{array}{l}1.572 \text { niños sanos } \\
324 \text { niños con FR }\end{array}$ & $\begin{array}{l}8 \text { y } 18 \\
\text { meses }\end{array}$ & $\begin{array}{l}\text { Consistencia interna } \\
\text { Estabilidad temporal } \\
\text { Acuerdo interjuez }\end{array}$ & $\begin{array}{l}\text { Validez de concurrente } \\
\text { Validez de constructo }\end{array}$ \\
\hline
\end{tabular}

${ }^{*}$ ND no determinado. ${ }^{* *}$ cuestionarios completados por educadores.

dación coinciden en que las propiedades psicométricas del ASQ lo hacen apropiado para su aplicación masiva (tabla 2) ) $^{33-41}$.

Este cuestionario se ha implementado en distintos escenarios como el domicilio, salas de espera, on line o por correo electrónico ${ }^{8}$. Parecería que su aplicación en las sala de espera no equipada como para evaluar las actividades del niño, podría ser menos confiable, como ocurrió en el estudio de Ryds ${ }^{42}$; Guevara demostró que no hay diferencia en la eficacia del ASQ al ser completado con o sin asistencia profesional ${ }^{19}$. Por otro lado, en la validación realizada en Brasil, fueron las educadoras quienes lo aplicaron $^{41}$. Aún no existe evidencia suficiente para determinar cómo estos contextos influyen en la confiabilidad de las respuestas.

Otros aspectos en discusión incluyen la definición de riesgo de déficit del DSM según ASQ; mientras algunos autores recomiendan considerar el puntaje total, otros son partidarios de basarse en los dominios, catalogando como en riesgo de déficit a un niño que tenga al menos un área descendida, aunque otros autores consideran dos o más dominios bajo los puntos de corte (tabla 3) 32,37,38,43. Por otro lado, sería interesante conocer el aporte de la dimensión cualitativa del cuestionario. Deakin-Bell mostró que las preocupaciones de los padres acerca de la salud o desarrollo de los niños, expresadas en las preguntas abiertas del ASQ, se relacionaban con un menor rendimiento en el Bayley-III ${ }^{44}$. Por otro lado, Horman demostró que, al incorporar la dimensión de preguntas abiertas en el cuestionario de los 5 años, aumenta la sensibilidad pero disminuye la es- 
Tabla 3 Sensibilidad y especificidad del ASQ según distintos estudios

\begin{tabular}{|c|c|c|c|c|c|c|c|}
\hline Autores & $\begin{array}{l}\text { Lugar } \\
\text { Año }\end{array}$ & Población & $\begin{array}{c}\text { Edad } \\
\text { aplicación }\end{array}$ & Referencia & Criterio déficit & $\begin{array}{l}\text { Sensibili- } \\
\text { dad \% }\end{array}$ & $\begin{array}{l}\text { Especifici- } \\
\text { dad } \%\end{array}$ \\
\hline $\begin{array}{l}\text { Squires \& } \\
\text { Bricker }^{9}\end{array}$ & $\begin{array}{l}\text { USA } \\
2009\end{array}$ & $\begin{array}{l}579 \text { niños: } \\
44 \% \text { con déficit del } \\
\text { desarrollo }\end{array}$ & $\begin{array}{l}2 \text { a } 60 \\
\text { meses }\end{array}$ & $\left.\mathrm{BD}\right|^{*}$ & 1 o más dominios & 86 & 85 \\
\hline $\begin{array}{l}\text { Skellern } \\
\text { y cols }{ }^{58}\end{array}$ & $\begin{array}{c}\text { Australia } \\
2001\end{array}$ & 136 RNPT extremos & $\begin{array}{c}12,18,24 \text { y } \\
48 \text { meses }\end{array}$ & $\begin{array}{l}\text { Varios } \\
\text { instrumentos }\end{array}$ & 1 o más dominios & 90 & 77 \\
\hline $\begin{array}{l}\text { Rydz } \\
y \text { cols }^{42}\end{array}$ & $\begin{array}{l}\text { Canada, } \\
2006\end{array}$ & $\begin{array}{l}101 \text { niños de } \\
\text { término sin factores } \\
\text { de riesgo }\end{array}$ & 18 meses & $\left.\mathrm{BD}\right|^{*}$ & 1 o más dominios & 67 & 39 \\
\hline $\begin{array}{l}\text { Kim \& } \\
\text { Sung }^{59}\end{array}$ & $\begin{array}{l}\text { Korea, } \\
2007\end{array}$ & 67 RNT & $\begin{array}{l}6 \text { a } 30 \\
\text { meses }\end{array}$ & BSID-II** & 1 o más dominios & 50 & 78 \\
\hline $\begin{array}{l}\text { Yu } \\
\text { y cols }\end{array}$ & $\begin{array}{l}19 \text { países, } \\
2007\end{array}$ & $\begin{array}{l}828 \text { niños: } \\
\text { 30\% RNPT }\end{array}$ & $\begin{array}{l}4 \text { a } 60 \\
\text { meses }\end{array}$ & $\begin{array}{l}\text { Distintos } \\
\text { instrumentos }\end{array}$ & 1 o más dominios & 87 & 82 \\
\hline $\begin{array}{l}\text { Lindsay } \\
y \text { cols }^{28}\end{array}$ & $\begin{array}{c}\text { Australia } \\
2008\end{array}$ & $\begin{array}{l}55 \text { niños con Ence- } \\
\text { falopatía hipóxico } \\
\text { Isquémica }\end{array}$ & $\begin{array}{l}6 \text { y } 12 \\
\text { meses }\end{array}$ & BSID-II** & 1 o más dominios & 67 & 93 \\
\hline $\begin{array}{l}\text { Kerstjens } \\
\text { y cols }\end{array}$ & $\begin{array}{l}\text { Holanda } \\
2009\end{array}$ & $\begin{array}{l}562 \text { RNT } \\
1510 \text { RNPT }\end{array}$ & 48 meses & $\begin{array}{l}\text { Necesidad } \\
\text { de educación } \\
\text { especial }\end{array}$ & 1 o más dominios & 89 & 80 \\
\hline $\begin{array}{l}\text { Gollenberg } \\
\text { y cols }\end{array}$ & $\begin{array}{l}\text { USA } \\
2009\end{array}$ & 40 RNT & 24 meses & BSID-II** & 1 o más dominios & 39 & 97 \\
\hline $\begin{array}{l}\text { Juneja } \\
\text { y cols }\end{array}$ & $\begin{array}{l}\text { India } \\
2011\end{array}$ & $\begin{array}{l}200 \text { niños: } \\
40 \% \text { con factores } \\
\text { de riesgo biológicos }\end{array}$ & $\begin{array}{l}4,10,18 y \\
24 \text { meses }\end{array}$ & $\begin{array}{l}\text { DAS III (basado } \\
\text { en BSID }{ }^{* *} \text { ) }\end{array}$ & 1 o más dominios & 83 & 75 \\
\hline $\begin{array}{l}\text { Limbos } \\
y \operatorname{cols}^{32}\end{array}$ & $\begin{array}{l}\text { Canada } \\
2011\end{array}$ & 334 RNT & $\begin{array}{l}12 \text { a } 60 \\
\text { meses }\end{array}$ & $\begin{array}{l}\text { Bayley-III*** } \\
\text { y otros } \\
\text { instrumentos }\end{array}$ & 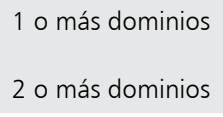 & $\begin{array}{l}82 \\
47\end{array}$ & $\begin{array}{l}78 \\
94\end{array}$ \\
\hline $\begin{array}{l}\text { Kapci } \\
\text { y cols }\end{array}$ & $\begin{array}{l}\text { Turquía } \\
2011\end{array}$ & $\begin{array}{l}833 \text { niños: } \\
28 \% \text { con factores } \\
\text { de riesgo } \\
4 \% \text { con déficit del } \\
\text { desarrollo }\end{array}$ & $\begin{array}{l}4 \text { a } 60 \\
\text { meses }\end{array}$ & $\begin{array}{l}\text { Varios } \\
\text { instrumentos }\end{array}$ & $\begin{array}{l}1 \text { o más dominios } \\
2 \text { o más dominios }\end{array}$ & $\begin{array}{l}99 \\
94\end{array}$ & $\begin{array}{l}58 \\
86\end{array}$ \\
\hline $\begin{array}{l}\text { Flamant } \\
\text { y cols }{ }^{43}\end{array}$ & $\begin{array}{c}\text { Francia } \\
2011\end{array}$ & 703 RNPT & 24 meses & Brunet Lezine & $\begin{array}{l}1 \text { o más dominios } \\
2 \text { o más dominios } \\
\text { Score total }\end{array}$ & $\begin{array}{l}88 \\
60 \\
85\end{array}$ & $\begin{array}{l}57 \\
82 \\
72\end{array}$ \\
\hline $\begin{array}{l}\text { Schonhaut } \\
\text { y cols }\end{array}$ & $\begin{array}{l}\text { Chile } \\
2013\end{array}$ & $\begin{array}{l}119 \text { RNT } \\
187 \text { RNPT }\end{array}$ & $\begin{array}{l}8,18 \text { y } 30 \\
\text { meses }\end{array}$ & Bayley $-I I{ }^{* * *}$ & 1 o más dominios & 73 & 81 \\
\hline $\begin{array}{l}\text { Halbwachs } \\
\text { y cols }\end{array}$ & $\begin{array}{c}\text { Francia } \\
2013\end{array}$ & 445 RNPT & 60 meses & WPPSI-III ${ }^{* * * *}$ & Score total & 80 & 54 \\
\hline $\begin{array}{l}\text { Hornman } \\
\text { y cols }{ }^{37}\end{array}$ & $\begin{array}{l}\text { Holanda } \\
2013\end{array}$ & $\begin{array}{l}394 \text { RNT } \\
1.063 \text { RNPT }\end{array}$ & 60 meses & $\begin{array}{l}\text { Necesidad } \\
\text { de educación } \\
\text { especial }\end{array}$ & $\begin{array}{l}1 \text { o más dominios } \\
\text { Score total }\end{array}$ & $\begin{array}{l}96 \\
88\end{array}$ & $\begin{array}{l}78 \\
93\end{array}$ \\
\hline $\begin{array}{l}\text { Armijo } \\
y \text { cols }^{46}\end{array}$ & $\begin{array}{l}\text { Chile } \\
2014\end{array}$ & $\begin{array}{l}1896 \text { niños: } \\
12 \% \text { RNPT } \\
5 \% \text { con déficit del } \\
\text { desarrollo }\end{array}$ & $\begin{array}{l}8 \text { y } 18 \\
\text { meses }\end{array}$ & Criterio clínico & 1 o más dominios & 94 & 80 \\
\hline
\end{tabular}

RNT Recién nacidos de término. RNPT recién nacidos prematuros. *BDI- Inventario de Desarrollo de Battelle. **BSID-II Escala de Bayley para el desarrollo infantil- $2^{\circ}$ edición. ${ }^{* *}$ Bayley -III Escala de Bayley para el desarrollo infantil del lactante y preescolar- $3^{\circ}$ edición. ****WPPSI-III Escala de inteligencia de Wechsler para niños-tercera edición. 
pecificidad del ASQ para predecir problemas escolares o necesidad de educación especial ${ }^{37}$. El definir estos aspectos sin duda nos llevará aún mucha discusión, y la definición se deberá basar en el contexto clínico y sensibilidad/ especificidad esperada para la pesquisa de los niños en riesgo de presentar dificultades en su desarrollo.

Considerando las cualidades descritas del ASQ y la tendencia a su validación a nivel mundial, nos preguntamos si era aplicable a nuestra población.

\section{Validación nacional ASQ}

Con el propósito de contar con un instrumento estandarizado para el tamizaje del DSM, que pueda ser usado transversalmente a nivel nacional, entre los años 2008 y 2011 se realizó una adaptación a la lingüística chilena del ASQ-3, para las edades de 8 y 18 meses. En dicho estudio, se incorporaron 1.896 niños atendidos en distintos Centros del Sistema público y privado de Atención de Salud.

La elección de un estándar de referencia fue un desafío; estudios de validación de instrumentos de tamizaje recomiendan aplicar un set de pruebas diagnósticas, no obstante, dado el alto costo y tiempo requerido para ello, no era una opción factible para nuestra realidad. Por este motivo, en la validación nacional del ASQ optamos por el criterio clínico y en una submuestra aplicamos la $3^{\circ}$ edición del Bayley

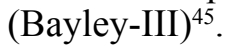

Al comparar los puntajes medios de la muestra chilena con la versión original del ASQ-3, destacaron diferencias significativas sólo en las áreas de motricidad gruesa a los 8 meses y resolución de problemas a los 18 meses, no obstante, se encontraron diferencias en los puntos de corte efectivos (tabla 4). Al analizar los puntos de corte mediante curvas de ROC, pudimos observar que la muestra chilena se ajustó bastante bien a los puntos de corte publicados en el manual del ASQ-3 (tablas 5 y 6). En ese sentido, la sensibilidad y especificidad del ASQ para la pesquisa del posible déficit del DSM, tomando como referencia el criterio clínico, fueron elevadas, 94\% y $80 \%$ respectivamente, la razón de verosimilitud positiva fue moderada, de 4,7 , mientras que la negativa fue buena, de $0,08^{46}$.

En un escenario más clínico, en que medimos la validez concurrente del ASQ tomando como referencia el Bayley-III,47 la sensibilidad y especificidad fueron de $73 \%$ y $81 \%$ respectivamente, valores apropiados para una prueba de screening del $\operatorname{DSM}^{7}$ y comparables con otros estudios (tabla 3$)^{28,32,48}$. Se ha reportado que las propiedades psicométricas del test aumentan a mayor edad de los niños evaluados y en poblaciones de mayor riesgo biológico de déficit $^{39,43,47,49}$, probablemente debido a mayor sensibilización de los padres.

La gran discusión que enfrentamos al validar una prueba de tamizaje es la efectividad versus la sobre-identificación de los niños en riesgo de déficit.

\section{Efectividad versus sobre-identificación en los programas de tamizaje del DSM}

Se ha demostrado que al implementar escalas estandarizadas para el tamizaje del de-

Tabla 4. Media, Desviación Estándar y puntos de corte efectivos del ASQ, comparación entre puntajes chilenos y de USA

\begin{tabular}{|lccccccc|}
\hline Escala & \multicolumn{2}{c}{$\begin{array}{c}\text { Chile } \\
\text { M (DS)-Cutoff }\end{array}$} & $\begin{array}{c}\text { ASQ } 8 \\
\text { M (DS)-Cutoff }\end{array}$ & $\begin{array}{c}\text { USA } \\
\text { M (DS)-Cutoff }\end{array}$ & $\begin{array}{c}\text { Chile } \\
\text { M (DS)-Cutoff }\end{array}$ \\
\hline Comunicación & $53,38(7,65)-37,9$ & 52,4 & $(9,67)-33,1$ & $39,33(12,79)-13,7$ & 42,3 & $(14,62)-13,1$ \\
\hline Motricidad gruesa & $44,82(12,73)-19,5$ & 52,09 & $(10,74)^{*}-30,6$ & 56,74 & $(5,77)-45,1$ & 55,46 & $(9,04)-37,4$ \\
\hline Motricidad fina & 54,85 & $(7,88)-39,1$ & 55,75 & $(7,8)-40,2$ & $50,72(9,58)-33,5$ & 52,44 & $(9,06)-34,3$ \\
Resolución de problemas & 54,74 & $(7,26)-40,4$ & 53,92 & $(8,87)-36,2$ & $47,34(10,07)-27,1$ & $45,99(10,13)-25,7$ \\
\hline Personal social & 52,19 & $(9,19)-33,8$ & 53,35 & $(8,75)-35,8$ & $51,59(7,88)-35,8$ & 47,9 & $(10,35)^{*}-27,2$ \\
\hline
\end{tabular}

Notas: Puntos de corte consideran promedio -2DS. *indican diferencia estadística entre puntajes chilenos y de USA $(p<0,05)$. 
Tabla 5. Sensibilidad y especificidad del ASQ, comparando puntos de cortes efectivos ASQ-3 y ASQ-Chile, para los 8 meses

\begin{tabular}{|c|c|c|c|c|c|c|}
\hline \multirow{3}{*}{ Escala } & \multicolumn{6}{|c|}{8 meses } \\
\hline & & Chile & & & USA & \\
\hline & $\begin{array}{l}\text { punto } \\
\text { corte }\end{array}$ & $\begin{array}{l}\text { Sensibilidad } \\
\text { (IC 95) }\end{array}$ & $\begin{array}{l}\text { Especificidad } \\
\text { (IC 95) }\end{array}$ & $\begin{array}{l}\text { punto } \\
\text { corte }\end{array}$ & $\begin{array}{l}\text { Sensibilidad } \\
\text { (IC 95) }\end{array}$ & $\begin{array}{l}\text { Especificidad } \\
\text { (IC 95) }\end{array}$ \\
\hline Comunicación & 35 & $0,49(0,37-0,60)$ & $0,96(0,94-0,97)$ & 30 & $0,46(0,34-0,57)$ & $0,98(0,97-0,99)$ \\
\hline Motricidad gruesa & 15 & $0,53(0,41-0,64)$ & $0,97(0,95-0,98)$ & 30 & $0,86(0,77-0,93)$ & $0,82(0,8-0,85)$ \\
\hline Motricidad fina & 35 & $0,66(0,54-0,77)$ & $0,95(0,94-0,97)$ & 40 & $0,76(0,66-0,86)$ & $0,9 \quad(0,88-0,92)$ \\
\hline Resolución de problemas & 40 & $0,76(0,66-0,86)$ & $0,9 \quad(0,89-0,92)$ & 35 & $0,66(0,54-0,77)$ & $0,95(0,94-0,97)$ \\
\hline Personal Social & 30 & $0,7 \quad(0,59-0,8)$ & $0,95(0,94-0,97)$ & 35 & $0,74(0,64-0,84)$ & $0,93(0,91-0,95)$ \\
\hline
\end{tabular}

Tabla 6. Sensibilidad y especificidad del ASQ, comparando puntos de cortes efectivos ASQ-3 y ASQ-Chile, para los 18 meses

\begin{tabular}{|c|c|c|c|c|c|c|}
\hline \multirow{3}{*}{ Escala } & \multicolumn{6}{|c|}{18 meses } \\
\hline & \multicolumn{3}{|c|}{ Chile } & \multicolumn{3}{|c|}{ USA } \\
\hline & $\begin{array}{l}\text { punto } \\
\text { corte }\end{array}$ & $\begin{array}{l}\text { Sensibilidad } \\
\text { (IC 95) }\end{array}$ & $\begin{array}{l}\text { Especificidad } \\
\text { (IC 95) }\end{array}$ & $\begin{array}{l}\text { punto } \\
\text { corte }\end{array}$ & $\begin{array}{l}\text { Sensibilidad } \\
\text { (IC 95) }\end{array}$ & $\begin{array}{l}\text { Especificidad } \\
\text { (IC 95) }\end{array}$ \\
\hline Comunicación & 10 & $0,68(0,53-0,8)$ & $0,98(0,98-0,99)$ & 10 & $0,68(0,53-0,83)$ & $0,98(0,98-0,99)$ \\
\hline Motricidad gruesa & 45 & $0,78(0,65-0,9)$ & $0,93(0,91-0,95)$ & 35 & $0,68(0,52-0,83)$ & $0,99(0,98-0,99)$ \\
\hline Motricidad fina & 30 & $0,73(0,6-0,85)$ & $0,95(0,93-0,96)$ & 30 & $0,73(0,6-0,85)$ & $0,95(0,93-0,96)$ \\
\hline Resolución de problemas & 25 & $0,63(0,48-0,78)$ & $0,95(0,94-0,97)$ & 25 & $0,63(0,48-0,78)$ & $0,95(0,94-0,97)$ \\
\hline Personal social & 35 & $0,7 \quad(0,55-0,85)$ & $0,95(0,93-0,96)$ & 25 & $0,63(0,48-0,78)$ & $0,99(0,99-1)$ \\
\hline
\end{tabular}

sarrollo en los programas de supervisión de salud, aumenta significativamente la pesquisa y derivación de niños con sospecha de déficit del DSM ${ }^{19,50}$. La pregunta que nos hacemos es cuántos de esos niños están siendo sobre-identificados y sobre-derivados y cuáles podrían ser los efectos negativos de dicha práctica.

Para conocer el valor predictivo del test frente a un paciente en particular (probabilidad post-test), es importante considerar la razón de probabilidad y la prevalencia (probabilidad pre-test). Así, con los valores obtenidos en la validación chilena, podemos decir que cuando la prevalencia de déficit es baja, la probabilidad post-test de déficit del DSM, dado un resultado positivo, es cercana al $50 \%$, y la probabilidad de déficit dado un resultado negativo, es de $1 \%$, lo que nos permite prácticamente descartar el déficit con un screening negativo. Por el contrario, en grupos de prematuros extremos, en que se podría esperar que prácticamente un tercio de los niños presenten dificultades del
DSM, la probabilidad post test de un resultado positivo aumenta a $70 \%$ y un resultado negativo sigue descartando prácticamente el déficit. (tabla 7).

Tradicionalmente se ha aceptado que un test de Screening del DSM sobre-pesquise entre un 15 a $30 \%$ de niños ${ }^{51}$. Si bien la sobre identificación se ha asociado con mayor (e innecesaria) ansiedad de los padres y efecto etiquetado, como ocurre en los Screening de enfermedades crónicas complejas ${ }^{52,53}$, en el caso del DSM, la consecuencia se relaciona con una mayor dedicación y estimulación del niño. Se ha evidenciado que los niños con tamizaje falsamente positivos, a largo plazo van a requerir mayor estimulación y apoyo en comparación con los verdaderos negativos ${ }^{54,55}$.

Para evitar una ansiedad innecesaria, parece importante involucrar y explicar a los padres correctamente los alcances y características de un proceso de screening, favoreciendo su colaboración y participación activa. Teóricamente, 
Tabla 7. Valores predictivos del ASQ considerando valores de sensibilidad 0,94 , especificidad 0,8 , LR+ 4,7 y LR- 0,08

\begin{tabular}{|lccc|}
\hline Escenario clínico & $\begin{array}{c}\text { Prevalencia } \\
\text { esperada }\end{array}$ & $\begin{array}{c}\text { Probabilidad } \\
\text { post-test dado un } \\
\text { resultado positivo }\end{array}$ & $\begin{array}{c}\text { Probabilidad } \\
\text { post- test dado un } \\
\text { resultado negativo }\end{array}$ \\
\hline Control sano & $15 \%$ & $45 \%$ & $1 \%$ \\
\hline Programa seguimiento prematuros & $33 \%$ & $70 \%$ & $4 \%$ \\
\hline $\begin{array}{l}\text { Seguimiento de niños con patología neurólogica o } \\
\text { genética que se asocia a déficit del DSM/discapacidad }\end{array}$ & $85 \%$ & $96 \%$ & $30 \%$ \\
\hline
\end{tabular}

uno de los efectos del uso de instrumentos de reporte parental es promover un insight derivado de la observación del DSM de sus hijos $^{27,56,57}$ por este motivo, considerando que el efecto "secundario" del tamizaje del DSM con ASQ podría ser ventajoso.

\section{Conclusiones}

Las alteraciones del DSM son frecuentes en la población infantil, considerando los beneficios del diagnóstico precoz e intervención temprana, la evaluación dirigida y sistemática del DSM debe ser incorporada en los controles de supervisión de salud. En vista de las propiedades técnicas y clínicas del ASQ, sumado a la factibilidad de su aplicación tanto en Atención Primaria como en las consultas de pediatría ambulatoria, y el posible efecto positivo de involucrar a los padres, pensamos que su implementación a nivel nacional sería un gran aporte para avanzar hacia los estándares de calidad mundialmente recomendados.

\section{Referencias}

1.- Gallaher MM, Christakis DA, Connell FA: Health care use by children diagnosed as having developmental delay. Arch Pediatr Adolesc Med 2002; 156 (3): 246-51.

2.- Boulet SL, Boyle CA, Schieve LA: Health care use and health and functional impact of developmental disabilities among US children, 1997-2005. Arch Pediatr Adolesc Med 2009; 163 (1): 19-26.

3.- Guralnick MJ: Effectiveness of early intervention for vulnerable children: a developmental perspective. Am J Ment Retard 1998; 102: 319-45.

4.- Anderson LM, Shinn C, Fullilove MT, et al: The effec- tiveness of early childhood development programs. Am J Prev Med 2003; 24 (3): 32-46.

5.- Schonhaut L, Álvarez J, Salinas P: El pediatra y la evaluación del desarrollo psicomotor. Rev Chil Pediatr 2008; 79 (supl 1): 26-31.

6.- Sheldrick RC, Merchant S, Perrin EC: Identification of Developmental-Behavioral Problems in Primary Care: A Systematic Review. Pediatrics 2011; 128 (2): 356-63.

7.- American Academy of Pediatrics, Council on Children With Disabilities, Section on Developmental Behavioral Pediatrics, Bright Futures Steering Committee, Medical Home Initiatives For Children With Special Needs Project Advisory Committee: Identifying infants and young children with developmental disorders in the medical home: an algorithm for developmental surveillance and screening. Pediatrics 2006; 118 (1): 405-20.

8.- Squires J, Bricker D, Potter L: Ages and stages questionnaires user's guide, 2nd edition. 1999. Baltimore: Paul Brookes.

9.- Squires J, Bricker D: Ages \& Stages Questionnaires. Third Edition. (ASQ-3): A Parent-Completed ChildMonitoring System. 2009. Stanford: Paul Brookes Publishing Company.

10.- Normas técnicas de evaluación y estimulación del desarrollo psicomotor en el niño y la niña menor de 6 años. Ministerio de Salud: Santiago Chile 2004. [accedido 26 de enero de 2014] disponible en http://www.crececontigo.gob.cl/wp-content/uploads/2013/06/Normas_tecnicas_estimulacion_y_evaluacion_desarrollo.pdf

11.- Schonhaut L, Maggiolo M, De Barbieri Z, Rojas $P$, Salgado A: Dificultades de lenguaje en preescolares: concordancia entre el test TEPSI y la evaluación fonoaudiológica. Rev Chil Pediatr 2007; 78 (4): 369-75.

12.- Vericat A, Orden AB: Herramientas de Screening del Desarrollo Psicomotor en Latinoamérica. Rev Chil Pediatr 2010; 81(5): 391-401.

13.- Registros estadísticos mensuales, DEIS-MINSAL 2012 http://chccsalud.cl/indicadores/chcc/consultas/ 
14.- Bedregal P: Hacia la renovación en las políticas de infancia en Chile. Rev Chil Pediatr 2014; 85 (1): 7-11.

15.- Subsecretaría de Salud Pública División de Planificación Sanitaria: II Encuesta de Calidad de Vida y Salud, Chile 2006. Disponible en: http://epi.minsal.cl/epi/html/ sdesalud/calidaddevida2006/Informe $\% 20$ Final $\% 20$ Encuesta $\% 20 \mathrm{de} \% 20 \mathrm{Calidad} \% 20 \mathrm{de} \% 20 \mathrm{Vida} \% 20 \mathrm{y} \% 20$ Salud\%202006.pdf

16.- Rosenberg SA, Zhang D, Robinson CC: Prevalence of developmental delays and participation in early intervention services for young children. Pediatrics 2008; 121 (6): e1503-9.

17.- Corrigan N, Stewart M, Scott M, Fee F: Predictive value of preschool surveillance in detecting learning difficulties. Arch Dis Child 1996; 74: 517-21.

18.- Jee SH, Szilagyi M, Ovenshire C, et al: Improved detection of developmental delays among young children in foster care. Pediatrics 2010; 125: 282-9.

19.- Guevara JP, Gerdes M, Localio R, et al: Effectiveness of developmental screening in an urban setting. Pediatrics 2013; 131 (1): 30-7.

20.- Squires J, Nickel RE, Eisert D: Early detection of developmental problems: strategies for monitoring young children in the practice setting. J Dev Behav Pediatr 1996; 17: 420-7.

21.- Sharma A: Developmental examination: birth to 5 years. Archives of Disease in Childhood. Education and Practice Edition 2011; 96 (5): 162-75.

22.- Figueiras A, Puccini RF, Silva EMK, Pedromônico MRM: Avaliação das práticas e conhecimentos de profissionais da atenção primária à saúde sobre vigilância do desenvolvimento infantil. Cad Saúde Pública 2003; 19 (6): 1691-9

23.- Radecki L, Sand-Loud N, O'Connor KG, Sharp S, Olson LM: Trends in the use of standardized tools for developmental screening in early childhood: 2002-2009. Pediatrics 2011; 128 (1): 14-9.

24.- Sices L, Feudtner C, McLaughlin J, Drotar D, Williams $M$ : How do primary care physicians identify young children with developmental delays? A national survey. Journal of Developmental \& Behavioral Pediatrics 2003; 24 (6): 409-17.

25.- Sand N, Silverstein M, Glascoe FP, Gupta VB, Tonniges $T P$, O'Connor $K G$ : Pediatricians' reported practices regarding developmental screening: do guidelines work? Do they help?. Pediatrics 2005; 116 (1): 174-9.

26.- Schonwald A, Horan K, Huntington N: Developmental Screening: Is There Enough Time? Clinical Pediatrics 2009; 48 (6): 648-55

27.- Schonhaut L, Armijo I, Millán T, et al: Comparación entre la evaluación tradicional desarrollo psicomotor versus una prueba autoadministrada. Rev Chil Pediatr 2010; 81(6): 498-505.

28.- Lindsay N, Healy G, Colditz P, Lingwood B: Use of the Ages \& Stages Questionnaire to predict outcome after hypoxic-ischaemic encephalopathy in the neonate. Journal of Paediatrics and Child Health 2008; 44: 590-5.

29.- Klamer A, Lando A, Pinborg A, Greisen G: Ages \& Stages Questionnaire used to measure cognitive deficit in children born extremely preterm. Acta Paediatrica 2005; 94: 1327-9.

30.- Halbwachs M, Muller J-B, Nguyen The Tich S, et al: Usefulness of parent-completed ASQ for neurodevelopmental screening of preterm children at five years of age. PLoS One 2013; 8 (8): e71925.

31.- Glascoe FP: Parents' concerns about children's development: prescreening technique or screening test. Pediatrics 1997; 99: 522-8.

32.- Limbos MM, Joyce DP: Comparison of the ASQ and PEDS in screening for developmental delay in children presenting for primary care. J Dev Behav Pediatr 2011; 32 (7): 499-511.

33.- Tsai H, McClelland M: Adaptation of the 36-Month Ages and Stages Questionnaire in Taiwan: Results from a preliminary study. J Early Interv 2006; 28: 213-25.

34.- Richter J, Janson H: A validation study of the Norwegian version of the Ages and Stages Questionnaires. Acta Pædiatrica 2007; 96 (5): 748-52.

35.- Heo KH, Squires J, Yovanoff P: Cross-cultural adaptation of a pre-school screening instrument: comparison of Korean and US populations. J Intellect Disabil Res 2008; 52 (3): 195-206.

36.- Kerstjens $J M$, Bos AF, Ten Vergert EMJ, de Meer $G$, Butcher PR, Reijneveld SA: Support for the global feasibility of the Ages and Stages Questionnaire as developmental screener. Early Hum Dev 2009; 85 (7): 443-7.

37.- Hornman J, Kerstjens JM, de Winter AF, Bos AF, Reijneveld $S A$ : Validity and internal consistency of the Ages and Stages Questionnaire 60-month version and the effect of three scoring methods. Early Hum Dev 2013; 89 (12): 1011-5.

38.- Kapci EG, Kucuker S,Uslu RI: How Applicable Are Ages and Stages Questionnaires for Use With Turkish Children? Topics in Early Childhood Special Education 2010; 30 (3): 176-88.

39.- Juneja M, Mohanty M, Jain R, Ramji S: Ages and Stages Questionnaire as a Screening Tool for Developmental Delay in Indian Children. Indian Pediatrics 2012; 49 (6): 457-61. 
40.- Vameghi R, Sajedi F, Kraskian Mojembari A, Habiollahi A, Lornezhad HR, Delavar B: Cross-Cultural Adaptation, Validation and Standardization of Ages and Stages Questionnaire (ASQ) in Iranian Children. Iran J Publ Heal 2013; 42 (5): 522-8.

41.- Filgueiras A, Pires P, Maissonette S, Landeira-Fernandez J: Psychometric properties of the Brazilian-adapted version of the Ages and Stages Questionnaire in public child daycare centers. Early Hum Dev 2013; 89 (8): 561-76.

42.- Rydz D, Srour M, Oskoui M, et al: Screening for developmental delay in the setting of a community pediatric clinic: a prospective assessment of parent-report questionnaires. Pediatrics 2006; 118 (4): 1178-86.

43.- Flamant C, Branger B, Nguyen S, et al: Parent-Completed Developmental Screening in Premature Children: A Valid Tool for Follow-Up Programs. Plos ONE 2011; 6 (5): 4-9.

44.- Deakin-Bell N, Walker K, Badawi N: The accuracy of parental concern expressed in the Ages and Stages Questionnaire to predict developmental delay. J Paediatr Child Health 2013; 49 (2): E133-6.

45.- Bayley N: Bayley Scales of Infant and Toddler Development. San Antonio, TX: The Psychological Corporation. 2006.

46.- Armijo I, Schonhaut L, Cordero M: Validation of the Chilean version of the Ages and Stages Questionnaire in Community Health Settings. En prensa.

47.- Schonhaut L, Armijo I, Schonstedt M, Alvarez J, Cordero $M$ : Validity of the ages and stages questionnaires in full term and preterm infants. Pediatrics 2013; 131: e1468-75.

48.- Yu LM, Hey E, Doyle LW, et al: Evaluation of the Ages and Stages Questionnaires in identifying children with neurosensory disability in the Magpie Trial follow-up study. Acta Paediatrica 2007; 96 (12): 1803-8.

49.- Schonhaut L, Salinas P, Armijo I, Schonstedt M, Álvarez $J$, Manríquez M: Validación de un cuestionario autoadministrado para la evaluación del desarrollo psicomotor. Rev Chil Pediatr 2009; 80 (6): 513-9.

50.- Hix-Small H, Marks K, Squires J, Nickel R: Impact of Implementing Developmental Screening at 12 and 24 Months in a Pediatric Practice. Pediatrics 2007; 120 (2): 381-9.
51.- Glascoe FP, Byrne KE: The accuracy of three developmental screening tests. J Early Intervention 1993; 17 : 368-79.

52.- Tluczek A, Mischler E, Farrell PM, et al: Parents' knowledge of neonatal screening and response to falsepositive cystic fibrosis testing. J Dev Behav Pediatr 1992; 13: 181-6.

53.- Fyro K, Bodegard G: Four-year follow-up of psychological reactions to false positive screening tests for congenital hypothyroidism. Acta Paediatr Scand 1987; 76: 107-14.

54.- Glascoe F: Are Overreferrals on Developmental Screening Tests Really a Problem? Arch Pediatr Adolesc Med 2001; 155: 54-9..

55.- Cadman D, Chambers LW, Walter SD, Ferguson R, Johnston N, McNamee J: Evaluation of public health preschool child developmental screening: the process and outcomes of a community program. American Journal of Public Health 1987; 77 (1): 45-51.

56.- Schonwald A, Huntington N, Chan E, Risko W, Bridgemohan $C$ : Routine developmental screening implemented in urban primary care settings: more evidence of feasibility and effectiveness. Pediatrics 2009; 123 (2): 660-8.

57.- Sices L, Drotar D, Keilman A, Kirchner LH, Roberts $D$, Stancin T: Communication About Child Development During Well-Child Visits: Impact of Parents' Evaluation of Developmental Status Screener With or Without an Informational Video. Pediatrics 2008 122: e1091-9.

58. Skellern CY, Rogers Y, Callaghan MJO. A parentcompleted developmental questionnaire : Follow up of ex-premature infants. Community Health. 2001 37(2), 125-129.

59. Kim EY, Sung IK. The ages and stages questionnaire: screening for developmental delay in the setting of a pediatric outpatient clinic. Korean journal of pediatrics 2007; 50 (11): 1061-6.

60. Gollenberg a L, Lynch CD, Jackson LW, McGuinness BM, Msall ME. Concurrent validity of the parentcompleted Ages and Stages Questionnaires, 2nd Ed. with the Bayley Scales of Infant Development II in a low-risk sample. Child. Care. Health Dev 2010; 36 (4): 485-90. 